\title{
Bandpass Filter Based On Parallel Cascaded Multiple Microring Resonators
}

\author{
Freddy Susanto Tan, Henry Kelderman and Alfred Driessen \\ Integrated Optical MicroSystems (IOMS) Group, MESA ${ }^{+}$Research Institute, Faculty of Electrical \\ Engineering, Mathematics, and Computer Science, University of Twente \\ P.O.Box 217, 7500 AE Enschede, The Netherlands.
}

\begin{abstract}
A cascaded multiple microring resonators device exhibits a boxshape filter response and better isolation than a single microring resonator device. A parallel cascaded three microring resonator device has been realized in Silicon Oxynitride ( $\mathrm{SiON}$ ) technology to demonstrate functionality of the devices as a bandpass filter.
\end{abstract}

\section{INTRODUCTION}

A single microring resonator device with high wavelength selectivity has promising functionality as a filter. Its small size in combination with low on-chip insertion loss [1] makes it a promising candidate for building blocks of very-large scale integrated (VLSI) photonic circuits [2]. The Lorentzian shape of the filter response, however, makes them less suited for bandpass filter applications. A device consisting of cascaded multiple microring resonators exhibits a more suitable filter shape.

A cascaded three microring resonator in parallel configuration, which is vertically coupled to port waveguides, has been realized in SiON technology. The bandpass filter functionality is demonstrated by the response of the drop port.

\section{REALIZATION AND CHARACTERIZATION}

Fig. 1 presents the cross-section of the device with its dimensions and a picture of the realized device obtained with $50 \mathrm{X}$ microscope objective.

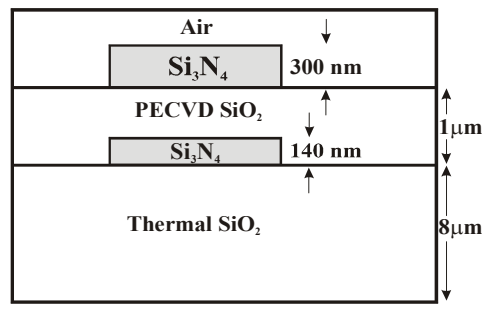

(a)

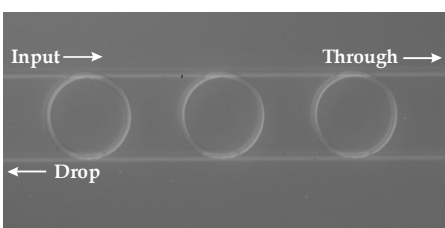

(b)

FIGURE 1. (a) Cross-section of the designed device with its dimensions; (b) picture of the realized device obtained with $50 \mathrm{X}$ microscope objective lens. 
Characterization has been done by launching TE polarized light from a tunable laser diode into the input port of the device, see Fig. 1b. The output from the drop port has been measured as a function of wavelength.

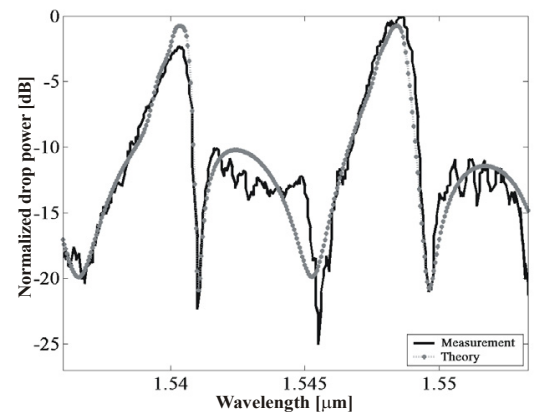

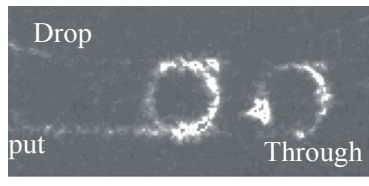

(b)

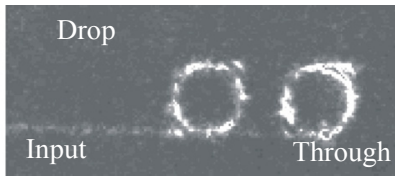

(c)

(a)

FIGURE 2. (a) drop response of a cascaded three microring resonators device, experimental data: solid black line; simulation with fitted parameters: gray dotted line. (b) Infra-red picture of a parallel cascaded device shows on-resonance (c) off-resonance.

The measurement result is presented in Fig.2a. Typical infra-red pictures taken from the top of a cascaded device are given in Fig. $2 b$ for on-resonance and Fig. $2 c$ for off- resonance. Fig. 2a shows a rejection ratio of $20 \mathrm{~dB}$ with a suppression of $10 \mathrm{~dB}$. By fitting the simulation model [3] to the experimental data in Fig. 2a, the propagation loss inside the microring resonator has been estimated to be $8 \mathrm{~dB} / \mathrm{cm}$ or 0.13 $\mathrm{dB} /$ roundtrip. Calculated bending loss [4] is $1 \mathrm{~dB} / \mathrm{cm}$. The absorption loss and sidewall roughness due to material imperfections resulting in scattering loss is estimated to be $7 \mathrm{~dB} / \mathrm{cm}$. The coupling coefficient is estimated to be 0.45 .

The filter performance can be largely enhanced by allowing different coupling coefficients for the individual resonators to the through as well as the drop ports. In the fabrication process it can be done by varying the lateral position of the ports with respect to the ring on the lithographic mask (see Fig. 1a for a ring with zero offset).

\section{ACKNOWLEDGMENTS}

This work has been carried out within the EC funded IST project NAIS, Nextgeneration Active Integrated-optics Subsystems, IST-2000-28018

\section{REFERENCES}

1. Tan, F. S., Klunder, D. J. W., Sengo, G., Kelderman, H., Hollink, A., Hoekstra, H. J. W. M., Driessen, A., Journal of Nonlinear Optical Physics and Materials, 12 (2), 205-212 (2003).

2. Little, B. E., Chu, S. T., Pan, W., Kokubun, Y., IEEE Photon. Technol. Lett., 12, 323-325 (2000).

3. Tan, F. S., Kelderman, H., Driessen, A., Proc. of ECOC, Rimini, Italy, 2003, pp. 364-365.

4. Selene Stress and Temperature, commercial product of $\mathrm{C} 2 \mathrm{~V}$. 